\title{
Predictors of visual and anatomical outcomes for neovascular age-related macular degeneration treated with bevacizumab
}

\author{
CHAORAN MA ${ }^{1 *}$, LIANG BAI $^{1 *}$, CHUNLING LEI $^{2}$, CHANGRUI WU $^{3}$, \\ QIANG SHI ${ }^{3}$, FENG HU ${ }^{1}$, ZHENXUAN HAO $^{1}$ and LE MA ${ }^{1}$ \\ ${ }^{1}$ Health Science Center, Xi'an Jiaotong University, Xi'an, Shaanxi 710061; ${ }^{2}$ The Fourth Hospital of Xi'an, \\ Xi'an Jiaotong University, Xi'an, Shaanxi 710004; ${ }^{3}$ The First Affiliated Hospital, \\ Xi'an Jiaotong University, Xi'an, Shaanxi 710061, P.R. China
}

Received December 18, 2014; Accepted February 20, 2015

DOI: $10.3892 /$ br.2015.448

\begin{abstract}
The present study aimed to evaluate the predictive factors for visual and anatomical outcomes in neovascular age-related macular degeneration (AMD) patients treated with intravitreal bevacizumab (IVB). A total of 113 patients with neovascular AMD received IVB treatment. The best corrected visual acuity (BCVA), central retinal thickness (CRT) and total macular volume (TMV) were assessed before the injection, and at 1,2, 3 and 9 months after surgery. Changes in BCVA and these optical coherence tomography (OCT) outcomes from baseline were compared, and independent predictors were evaluated by logistic regression models. During the treatment, logarithm of the minimum angle of resolution (logMAR) significantly decreased from 1.12 to 0.83 , and reductions in OCT parameters were earlier and larger. Baseline BCVA was associated with the changes in BCVA and CRT, whereas baseline OCT features significantly affected their own changes. Larger baseline $\operatorname{logMAR}$ and OCT features were more likely to experience a greater proportion of $\geq 50 \mu \mathrm{m}$ reduction in CRT $(\mathrm{P}<0.05)$. The BCVA decreases were positively associated with the reductions in CRT ( $\mathrm{r}=0.34, \mathrm{P}<0.01)$ and TMV $(\mathrm{r}=0.41, \mathrm{P}<0.01)$. Among patients with neovascular AMD, IVB resulted in earlier significant decreases in TMV and CRT, suggesting that these OCT anatomical outcomes may be considered as more sensitive responders to evaluate the treatment effects of bevacizumab.
\end{abstract}

\section{Introduction}

Age-related macular degeneration (AMD) is the leading cause of severe visual impairment and irreversible blindness in

Correspondence to: Dr Le Ma, Health Science Center, Xi'an Jiaotong University, 76 Yanta West Road, Xi'an, Shaanxi 710061, P.R. China

E-mail:male@mail.xjtu.edu.cn

${ }^{*}$ Contributed equally

Key words: visual performance, optical coherence tomography, neovascular age-related macular degeneration, bevacizumab, predictors individuals aged $>55$ years in developed countries. Choroidal neovascularization (CNV), the later stage of AMD, is associated with increased vascular permeability and angiogenesis, involving abnormal serous fluid collection at the central area of the retina that causes serious visual dysfunction (1). The prevalence of AMD was estimated as $8.7 \%$ in $>45$ year olds worldwide, while $7.0 \%$ of $\geq 65$ year olds had AMD in China. The total number of global AMD patients will rise sharply to 196 million in 2020 with increasing longevity. Therefore, the economic and social burden of AMD is becoming one of the crucial public health issues among an aging population (2).

Although the pathogenesis of AMD remains to be elucidated, upregulation of vascular endothelial growth factor (VEGF) appears to play a causal role in CNV. VEGF induces cell proliferation, survival, permeability, nitric oxide production, migration and tissue factor production. Inhibition of VEGF is a recommended treatment approach to neovascular AMD (3-5). As a humanized monoclonal antibody, bevacizumab could withdraw all the forms of VEGF, leading to cessation of vascular growth and neovascular regression by withdrawing or interfering with the function of VEGF. Several studies reported that intravitreal bevacizumab (IVB) was safe and effective for neovascular AMD; however, the efficacy varied substantially among different ethnicities (6). Thus far, the majority of associative research is specific to the Caucasian population. Additionally, epidemiological studies indicated that certain predictive factors may be associated with visual and anatomical outcomes following IVB, and studies specifically concerning the efficacy of IVB for different populations are limited $(7,8)$. Of note, the identification of these predictors should be warranted $(9,10)$.

Therefore, the purpose of the present study was to evaluate the predictive factors for visual and anatomical outcomes in Chinese neovascular AMD patients subsequent to receiving IVB treatment.

\section{Materials and methods}

Subjects. Potential participants were recruited from the intravitreal injection clinic at the First Affiliated Hospital of Xi'an Jiaotong University (Xi'an, China). The eligible participants were diagnosed with neovascular AMD, which was defined as 
retinal pigment epithelial detachment, serous sensory retinal detachment, subretinal hemorrhage or subretinal fibrosis observed in color photographs within 2 disc diameters of the macula center, or the application of photocoagulation for this disorder by the Age-Related Eye Disease Study (AREDS) Research Group.

Subjects were excluded if they had: i) The presence of extensive subfoveal fibrosis or retinal angiomatous proliferation, which may give a different response to anti-VEGF treatment; ii) other eye disorders including diabetic retinopathy, retinal detachment, significant cataract and glaucoma; iii) a history of vitrectomy; iv) prior treatment for $\mathrm{CNV}$, such as photodynamic therapy or any macular laser photocoagulation; v) uncontrolled systemic arterial hypertension or any vascular occlusive events (cardiovascular, cerebrovascular, or peripheral vascular accident); and vi) known coagulation abnormalities or current use of anticoagulative medication.

All the participants provided written informed consent prior to participation. The study was approved by the Institutional Review Board of Xi'an Jiaotong University.

Study design. At the baseline visit, all the subjects underwent a comprehensive ophthalmological examination, including slit-lamp evaluation, ophthalmoscopy, dilated-fundus photography, optical coherence tomography (OCT) examination, fundus fluorescein angiography (FFA) and visual function assessments [best corrected visual acuity (BCVA)]. Demographic characteristics and medical history were also recorded.

Qualified patients received the initial intravitreal injection under sterile conditions according to a standard protocol. Subsequent to povidone-iodine eye preparation, injection of $1.25 \mathrm{mg}(0.05 \mathrm{ml})$ bevacizumab (Avastin ${ }^{\mathrm{TM}}$; Genentech Inc., San Francisco, CA, USA) in a tuberculin syringe with a 30-gauge needle was performed through the pars plana, $3.5 \mathrm{~mm}$ posterior to the corneal limbus, which was anesthetized. Following the injection, intraocular pressure, retinal artery perfusion and light perception from all four directions were checked. Re-injections of bevacizumab were scheduled at least four weeks after the previous treatment. The decision to retreat at this stage was based on the appearance of the following criteria: i) Decline in BCVA by $>5$ letters at two repeated tests, as this decrease was associated with leakage on FFA or OCT; ii) recurrence of any subretinal fluid or cystic maculopathy on OCT in a previously dry macula; iii) increase of central retinal thickness (CRT) in OCT by $>100 \mu \mathrm{m}$; iv) appearance of new macular haemorrhage; and v) new area of classic CNV.

Patients were instructed to use topical ciprofloxacin (Ciloxan ${ }^{\mathrm{TM}}$; Alcon Laboratories, Inc., Puurs, Belgium) four times a day for three days. The patients were examined at one week after the injection and every month for one year. Any ocular complications or systemic events were monitored by ophthalmoscopic and physical examinations at each follow-up visit.

Outcome measures. BCVA, CRT and total macular volume (TMV) of each patient were measured before the injection and at 1, 2, 3 and 9 months after surgery. The same experienced ophthalmologist performed all the evaluations.

Following autorefraction (Topcon KR-8000; Topcon Corp., Tokyo, Japan), BCVA was assessed using Early
Treatment Diabetic Retinopathy Study charts at $4 \mathrm{~m}$ with an illuminated cabinet (Precision Vision, La Salle, IL, USA), and was transformed to the logarithm of the minimum angle of resolution ( $\log$ MAR) for statistical analysis. A significant improvement in CRT was defined as a reduction of $50 \mu \mathrm{m}$.

CRT and TMV were determined automatically by spectral-domain ocular coherence tomography (Cirrus high-definition OCT; Carl Zeiss Meditec, Inc., Dublin, CA, USA). Prior to starting the recording, the sitting position, place of the head, focus of the video image and centralization of the scan area were carefully adjusted. For this examination, the scanning was generated using the Macular Cube 512x128 scan pattern (128 horizontal lines each consisting of 512 A-scans/line) and the area of scanning was $6 \times 6 \mathrm{~mm}^{2}$. The images were regarded eligible if the retina was clearly visible and recognizable in each scan, without artifacts caused by eye movement and papillary shadowing during the recording, the full depth and extent of the retina was visualized and the scan signal strength was $>6 / 10$ in the machine. An intrinsic fixation light or, when the patient was unable to see it, an external fixation light was used $(11,12)$.

Statistical analysis. All the variables were tested for normal distribution with the Shapiro-Wilk test, and homogeneity of variances was evaluated by Levene's test. The proportions of participants, including demographic characteristics, systemic conditions, baseline vision acuity and OCT anatomical outcomes, were calculated respectively. Changes in BCVA, CRT and TMV from baseline of different subgroups were compared by independent Student's t-test or one-way analysis of variance for normal distributions, otherwise they were compared by the Wilcoxon rank test or the Kruskal-Wallis $\mathrm{H}$ test. Each predictor for gaining $>3$ lines in BCVA and a reduction of $>50 \mu \mathrm{m}$ in CRT were evaluated by logistic regression models. Correlations between the changes in BCVA and OCT anatomical outcomes were assessed using the Pearson test. All the data analyses were performed using the SPSS statistics software (SPSS version 17.0; SPSS, Inc., Chicago, IL, USA). All P-values were two-sided and $\mathrm{P}<0.05$ was considered to indicate a statistically significant difference.

\section{Results}

Participant characteristics. A total of 113 participants with a mean age of 68.3 years were enrolled in the study. These baseline characteristics are presented in Table I. In total, $44.2 \%$ were female, $29.2 \%$ were former or current cigarette smokers and $11.5 \%$ had a drinking history. During the 9-month treatment, no serious ocular or systemic event was noted; and none of the patients showed clinical evidence of intraocular inflammation, uveitis, endophthalmitis or signs of retinal toxicity. Certain patients complained once about blurred vision following the bevacizumab injection; however, this disappeared, usually within a few days. There was also no significant rise in blood pressure or intraocular pressure as recorded during the follow-up visits, and no thromboembolic events were observed during the study.

BCVA and OCT anatomical outcomes. The changes in BCVA from baseline to 9 months are shown in Fig. 1. LogMAR 
Table I. Characteristics of patients with neovascular AMD.

\begin{tabular}{lc}
\hline Characteristics & No. $(\%)$ \\
\hline Gender & \\
Male & $63(55.8)$ \\
Female & $50(44.2)$ \\
Age, years & \\
$<65$ & $38(33.6)$ \\
$65-75$ & $40(35.4)$ \\
$\geq 75$ & $35(31.0)$ \\
Systemic conditions & \\
Smoking history & $33(29.2)$ \\
Drinking history & $13(11.5)$ \\
Hypertension & $42(37.2)$ \\
BMI, kg/m ${ }^{2}$ & \\
$<24$, normal & $60(53.1)$ \\
$24-28$, overweight & $48(42.5)$ \\
$\geq 28$, obesity & $5(4.4)$ \\
BCVA, mean \pm SD & \\
logMAR & $1.12 \pm 0.53$ \\
OCT measurements, mean \pm SD & \\
CRT, $\mu$ m & $353.1 \pm 128.0$ \\
TMV, mm ${ }^{3}$ & $10.7 \pm 2.0$ \\
\hline A & \\
\hline
\end{tabular}

AMD, age-related macular degeneration; BCVA, best corrected visual acuity; BMI, body mass index; CRT, central retinal thickness; logMAR, logarithm of the minimum angle of resolution; OCT, optical coherence tomography; SD, standard deviation; TMV, total macular volume.

decreased from 1.12 to $1.04(\mathrm{P}=0.15)$ at 1 month, and later it experienced a gradually slow reduction to 0.83 at 9 months. Unlike the previous time-points, $\log$ MAR achieved significance at the final follow-up, compared to baseline $(\mathrm{P}=0.02)$. At this visit, 89 subjects (78.8\%) presented with improvements in BCVA.

Parallel to the change of $\log$ MAR, the magnitudes of reductions in OCT parameters were earlier and larger, particularly at the first month, when the decreases in CRT and TMV were significant $\left(\mathrm{P}_{\mathrm{CRT}}<0.01\right.$ and $\mathrm{P}_{\mathrm{TMV}}=0.02$, respectively). Following this, the OCT outcomes remained stable through the 9 months.

Predictors for changes of BCVA and OCT anatomical outcomes at 9 months. Univariate analysis was performed to verify whether there were any statistical associations of the changes in BCVA and OCT anatomical outcomes with baseline characteristics. Baseline BCVA was a significant predictor of changes in BCVA and CRT. Compared to baseline $\log$ MAR $<1$, subjects with baseline $\log$ MAR $\geq 1$ exhibited a greater $\log$ MAR and CRT change $(\mathrm{P}<0.05)$. In addition, individuals with a larger baseline OCT elevation tended to change less in BCVA, compared to those with mild impairment in fundus morphology.

For OCT anatomical outcomes, all the baseline features were associated with their own changes at 9 months. Individuals with baseline CRT $<400 \mu \mathrm{m}$ experienced $29.60 \mu \mathrm{m}$ and $0.35 \mathrm{~mm}^{3}$ reductions in CRT and TMV at
A

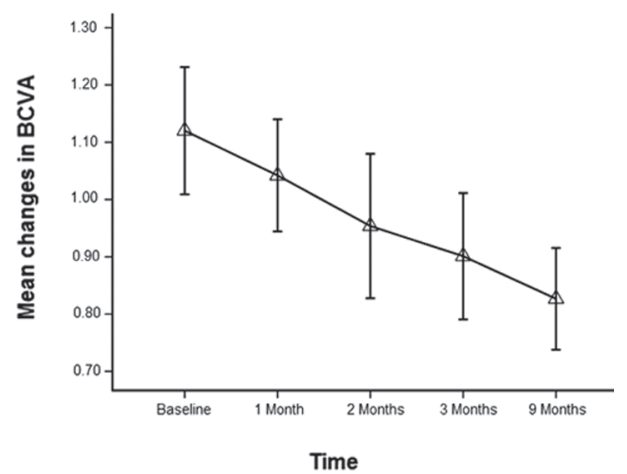

B

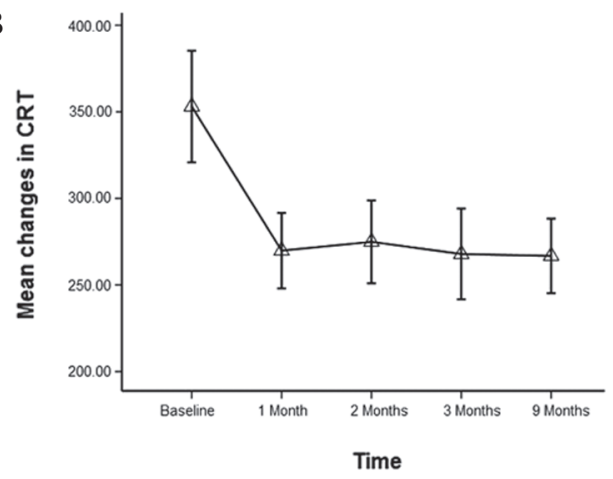

C

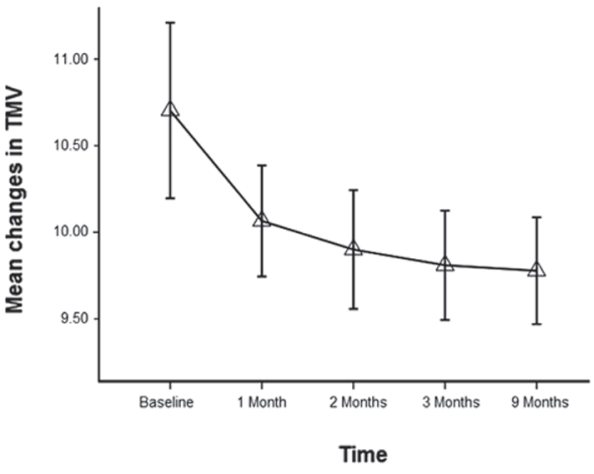

Figure 1. Mean change in (A) best corrected visual acuity (BCVA), (B) central retinal thickness (CRT) and (C) total macular volume (TMV) over 9 months in patients treated with intravitreal bevacizumab (IVB) for neovascular age-related macular degeneration (AMD). Data are presented as the mean. Vertical bars represent the $95 \%$ confidence interval.

9 months, while $199.67 \mu \mathrm{m}$ and $2.07 \mathrm{~mm}^{3}$ decreases were found in those with baseline CRT $\geq 400 \mu \mathrm{m} \quad(\mathrm{P}<0.01)$. Similarly, larger baseline TMV were associated with more substantial changes in OCT outcomes at 9 months $(\mathrm{P}<0.01)$. There were no statistically significant interactions between any of these predictors.

Furthermore, when baseline factors were considered simultaneously in the binary logistic regression (Table II), the proportion of $\geq 3$ lines gain in BCVA was lower in subjects with baseline $\log$ MAR $<1$ compared to those with baseline $\log$ MAR $\geq 1$ [odds ratio (OR), 4.84; 95\% confidence interval (CI), 1.73-13.58]. For the CRT decrease, both larger baseline logMAR (OR, 5.56; 95\% CI, 1.93-16.05) and OCT features $\left(\mathrm{OR}_{\mathrm{CRT}}, 57.00\right.$; 95\% CI, 12.48-260.43; and $\mathrm{OR}_{\mathrm{TMV}}, 9.47$; $95 \%$ CI, 3.31-27.13) were significantly more likely to appear in a greater proportion of $\geq 50 \mu \mathrm{m}$ CRT reduction $(\mathrm{P}<0.05)$. There were no statistically significant interactions between any of the described factors. 
Table II. Analysis for $\geq 3$-line gain in BCVA and $\geq 50 \mu \mathrm{m}$ reduction in CRT.

\begin{tabular}{|c|c|c|c|c|c|c|}
\hline Characteristics & $\begin{array}{l}\geq 3 \text {-line gain, } \\
\text { n/N }(\%)\end{array}$ & $\begin{array}{c}\text { OR } \\
(95 \% \mathrm{CI})\end{array}$ & P-value & $\begin{array}{l}\geq 50 \mu \mathrm{m} \mathrm{CRT} \\
\text { reduction, } \mathrm{n} / \mathrm{N}(\%)\end{array}$ & $\begin{array}{c}\text { OR } \\
(95 \% \mathrm{CI})\end{array}$ & P-value \\
\hline \multicolumn{7}{|l|}{ Gender } \\
\hline Male & $32 / 63(50.8)$ & 1 & \multirow[t]{2}{*}{0.74} & $31 / 63(49.2)$ & 1 & \multirow[t]{2}{*}{0.85} \\
\hline Female & $27 / 50(54.0)$ & $1.14(0.54-2.39)$ & & $23 / 50(46.0)$ & $0.88(0.42-1.85)$ & \\
\hline \multicolumn{7}{|l|}{ Age, years } \\
\hline$<65$ & $21 / 38(55.3)$ & 1 & \multirow[t]{3}{*}{0.57} & $21 / 38(55.3)$ & 1 & \multirow[t]{3}{*}{0.51} \\
\hline $65-75$ & $21 / 40(52.5)$ & $0.90(0.37-2.18)$ & & $19 / 40(47.5)$ & $0.73(0.30-1.79)$ & \\
\hline$\geq 75$ & $17 / 35(48.6)$ & $0.77(0.30-1.92)$ & & 22/35 (62.9) & $1.37(0.54-3.50)$ & \\
\hline \multicolumn{7}{|l|}{ Smoking } \\
\hline No & $39 / 80(48.8)$ & 1 & \multirow[t]{2}{*}{0.25} & $40 / 80(50.0)$ & 1 & \multirow[t]{2}{*}{0.11} \\
\hline Yes & $20 / 33(60.6)$ & $1.62(0.71-3.69)$ & & $22 / 33(66.7)$ & $2.00(0.86-4.66)$ & \\
\hline \multicolumn{7}{|l|}{ Drinking } \\
\hline No & $51 / 100(51.0)$ & 1 & \multirow[t]{2}{*}{0.08} & 49/100 (49.0) & 1 & \multirow[t]{2}{*}{0.17} \\
\hline Yes & 10/13 (76.9) & $3.20(0.83-12.34)$ & & 9/13 (69.2) & $2.34(0.68-8.10)$ & \\
\hline \multicolumn{7}{|l|}{ Hypertension } \\
\hline No & 35/71 (49.3) & 1 & \multirow[t]{2}{*}{0.86} & $33 / 71(46.5)$ & 1 & \multirow[t]{2}{*}{0.39} \\
\hline Yes & $20 / 42(47.6)$ & $0.94(0.44-2.01)$ & & $16 / 42(38.1)$ & $0.71(0.33-1.54)$ & \\
\hline \multicolumn{7}{|l|}{ BMI, $\mathrm{kg} / \mathrm{m}^{2}$} \\
\hline$<24$ & $29 / 60(48.3)$ & 1 & \multirow[t]{3}{*}{0.30} & $29 / 60(48.3)$ & 1 & \multirow[t]{3}{*}{0.71} \\
\hline $24-28$ & $28 / 48(58.3)$ & $1.50(0.70-3.22)$ & & 23/48 (47.9) & $0.98(0.46-2.10)$ & \\
\hline$\geq 28$ & $3 / 5(60.0)$ & $1.60(0.25-10.30)$ & & $3 / 5(60.0)$ & $0.64(0.14-2.93)$ & \\
\hline \multicolumn{7}{|c|}{ BCVA baseline, logMAR } \\
\hline$<1$ & $6 / 29(20.7)$ & 1 & \multirow[t]{2}{*}{$<0.01$} & 5/27 (18.5) & 1 & \multirow[t]{2}{*}{$<0.01$} \\
\hline$\geq 1$ & $53 / 84(63.1)$ & $4.84(1.73-13.58)$ & & $48 / 86(55.8)$ & $5.56(1.93-16.05)$ & \\
\hline \multicolumn{7}{|c|}{ CRT baseline, $\mu \mathrm{m}$} \\
\hline$<400$ & $23 / 38(60.5)$ & 1 & \multirow[t]{2}{*}{0.39} & $18 / 75(24.0)$ & 1 & \multirow[t]{2}{*}{$<0.01$} \\
\hline$\geq 400$ & $39 / 75(52.0)$ & $0.71(0.32-1.56)$ & & $36 / 38(94.7)$ & $57.00(12.48-260.43)$ & \\
\hline \multicolumn{7}{|c|}{ TMV baseline, $\mathrm{mm}^{3}$} \\
\hline$<10$ & 19/34 (55.9) & 1 & \multirow[t]{2}{*}{0.89} & $5 / 34(14.7)$ & 1 & \multirow[t]{2}{*}{$<0.01$} \\
\hline$\geq 10$ & $43 / 79(54.4)$ & $0.94(0.42-2.12)$ & & $49 / 79(62.0)$ & $9.47(3.31-27.13)$ & \\
\hline
\end{tabular}

BCVA, best corrected visual acuity; CRT, central retinal thickness; N, total no. of patients; OR, odds ratio; CI, confidence interval; BMI, body mass index; $\log$ MAR, logarithm of the minimum angle of resolution; TMV, total macular volume.

A

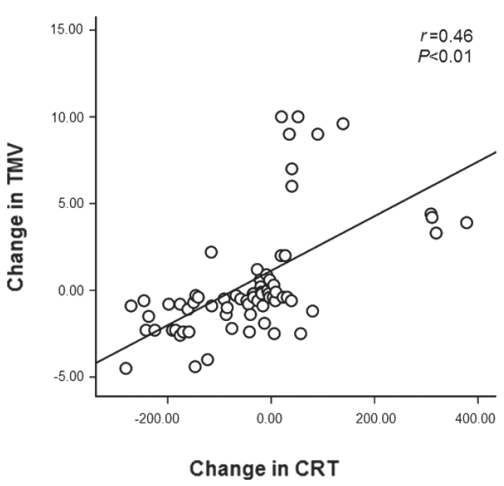

B

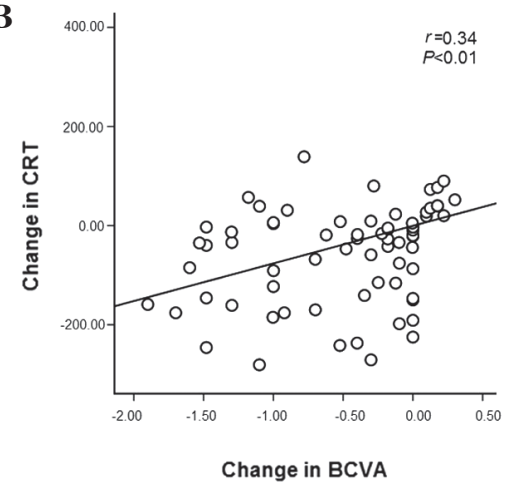

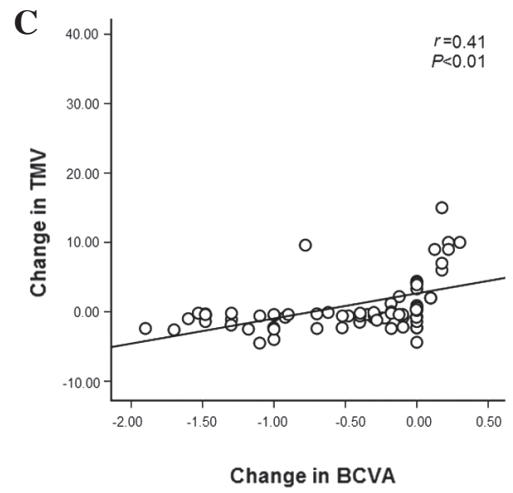

Figure 2. (A) Correlation between central retinal thickness (CRT) change and total macular volume (TMV) change ( $r=0.46, \mathrm{P}<0.01)$. (B) Correlation between best corrected visual acuity (BCVA) change and CRT change $(r=0.34, \mathrm{P}<0.01)$. (C) Correlation between BCVA change and TMV change $(r=0.41, \mathrm{P}<0.01)$.

Correlations between BCVA change and OCT anatomical outcome changes. In the correlation analysis, there was a significant association between CRT change and TMV change $(r=0.46, P<0.01)$. Positive linear correlations of BCVA change with CRT $(\mathrm{r}=0.34, \mathrm{P}<0.01)$ and TMV $(\mathrm{r}=0.41, \mathrm{P}<0.01)$ were also observed significantly (Fig. 2). 


\section{Discussion}

The effect of bevacizumab was assessed in patients with neovascular AMD undergoing IVB. The present findings showed earlier significant changes in CRT and TMV after the 9-month IVB treatment. Furthermore, the decreases in these OCT anatomical outcomes were accompanied by a reduction in $\log$ MAR levels, suggesting that TMV and CRT may be more sensitive responders to evaluate the treatment effects of bevacizumab.

In the majority of previous studies, IVB produced a significant improvement of visual function. In the CATT study, which included patients with neovascular AMD, treatment with intravitreal injections of ranibizumab or bevacizumab for 1 year resulted in equivalent significant effects on vision $(13,14)$. In a prospective, open-label, nonrandomized clinical study by Bashshur et al (15), eyes with neovascular AMD treated with IVB for $>2$ years had significant functional improvement compared to baseline. The present study indicated that $\log \mathrm{MAR}$ levels of BCVA decreased gradually during the intervention, and a statistically significant reduction in BCVA $\log$ MAR was noted among the neovascular AMD patients after 9 months of IVB treatment (15). The less significant BCVA improvement in our studies may, in part, be correlated with a relatively smaller sample size that was probably insufficient to detect the similar amount of BCVA change. Alternatively, a between-ethnicity genetic variation of VEGF would influence the visual function and pharmacokinetics, which may also be responsible for the differences in biological endpoints to intravitreal therapy in our study and previous studies. In contrast to the absent significant change in BCVA at the first month, much larger decreases in the OCT anatomical outcomes were observed in the present study, and subsequently the patients had stable CRT and TMV levels over the 9 -month period $(16,17)$. The present results were comparable to those reported by Cleary et al (18), in which individuals assigned to receive IVB significantly decreased these OCT anatomical characteristics and remained significantly lower at 9 months compared to the baseline. Therefore, IVB is an effective strategy for helping to improve the visual function and fundus morphology in neovascular AMD patients.

Notably, earlier changes in fundus morphology and features were detected in the central retina in neovascular AMD eyes receiving IVB treatment compared to BCVA. The present results indicated that these OCT anatomical outcomes were highly predictive of change in macular status and the prognosis of macular disorders. Abnormal fluid collection within or below the retina was common in advanced AMD patients, which could lead to macular edema and severe thickening of central retina, and eventually result in visual dysfunction $(19,20)$. Bevacizumab suppressed the activation of the intracellular tyrosine kinase, inhibited VEGF-induced cell proliferation and migration, and lead the macular change towards normalization in morphology $(21,22)$. Therefore, normalizing the morphological integrity of the macula was essential for optimizing functional recovery of vision in those with neovascular AMD (23). This was also in agreement with results from the present study that an association existed between visual function promotion and reduction in CRT and TMV levels. Furthermore, improvements in BCVA were more pronounced in those with mild structural impairment of the macula compared to participants with severe AMD. Photoreceptors were unable to perform irreversibility; and visual dysfunction could hardly be improved, particularly for late AMD, due to the vital impairment of those cells. Thus, early treatment of neovascular AMD limited the $\mathrm{CNV}$-induced photoreceptor damage to restore to normal visual function (24-26). As an important predictor in evaluating the prognosis of AMD, OCT anatomical outcomes, when measured timely, could be of significant relevance in clinical practice.

Potential limitations of the present study should be mentioned. First, the conclusions regarding predictors for BCVA and OCT outcomes were not favorable in the absence of a control group; however, it was considered unethical not to offer a treatment to patients with neovascular AMD. Furthermore, early significant OCT outcome changes from baseline may also provide an indication for the eventual conclusion following a proper evaluation approach. Second, the study had a relatively short follow-up of 9 months, so no exact statements can be concluded with regard to the long-term prognosis.

In conclusion, the study demonstrated that treatment with bevacizumab resulted in earlier significant changes in OCT anatomical outcomes among Chinese patients with neovascular AMD, suggesting that the change towards normalization in ocular morphology may underlie the improvement of visual function. Therefore, it indicated that OCT anatomical outcomes could be identified as more favorable indexes to provide potentially useful insights into defining the optimal treatment strategy and evaluation of prognosis for AMD. Further studies are essential to verify the results and generalize them in a larger AMD population.

\section{Acknowledgements}

The present study was supported by the Fundamental Research Funds for the Central Universities of China (grant no. xjj2012052) and the Natural Science Foundation of Shaanxi Province of China (grant no. 2013JQ4008).

\section{References}

1. Bourne RR, Stevens GA, White RA, et al; Vision Loss Expert Group: Causes of vision loss worldwide, 1990-2010: A systematic analysis. Lancet Glob Health 1: e339-e349, 2013.

2. Wong WL, Su X, Li X, Cheung CM, Klein R, Cheng CY and Wong TY: Global prevalence of age-related macular degeneration and disease burden projection for 2020 and 2040: A systematic review and meta-analysis. Lancet Glob Health 2: e106-e116, 2014.

3. Chakravarthy U, Harding SP, Rogers CA, Downes SM, Lotery AJ, Culliford LA and Reeves BC; IVAN study investigators: Alternative treatments to inhibit VEGF in age-related choroidal neovascularisation: 2-year findings of the IVAN randomised controlled trial. Lancet 382: 1258-1267, 2013.

4. Ferrara N, Mass RD, Campa C and Kim R: Targeting VEGF-A to treat cancer and age-related macular degeneration. Annu Rev Med 58: 491-504, 2007.

5. Duh EJ, Yang HS, Haller JA, et al: Vitreous levels of pigment epithelium-derived factor and vascular endothelial growth factor: Implications for ocular angiogenesis. Am J Ophthalmol 137: 668-674, 2004.

6. Gupta OP, Shienbaum G, Patel AH, Fecarotta C, Kaiser RS and Regillo CD: A treat and extend regimen using ranibizumab for neovascular age-related macular degeneration clinical and economic impact. Ophthalmology 117: 2134-2140, 2010. 
7. Jonas JB, Tao Y and Schlichtenbrede FC: Intravitreal bevacizumab for exudative age-related macular degeneration in clinical practice. J Ocul Pharmacol Ther 27: 467-470, 2011.

8. Yamashiro K, Tomita K, Tsujikawa A, Nakata I, Akagi-Kurashige Y, Miyake M, Ooto S, Tamura $\mathrm{H}$ and Yoshimura N: Factors associated with the response of age-related macular degeneration to intravitreal ranibizumab treatment. Am J Ophthalmol 154: 125-136, 2012.

9. Avery RL, Pieramici DJ, Rabena MD, Castellarin AA, Nasir MA and Giust MJ: Intravitreal bevacizumab (Avastin) for neovascular age-related macular degeneration. Ophthalmology 113: 363-372. $\mathrm{e} 5,2006$.

10. Mekjavic PJ, Kraut A, Urbancic M, Lenassi E and Hawlina M: Efficacy of 12-month treatment of neovascular age-related macular degeneration with intravitreal bevacizumab based on individually determined injection strategies after three consecutive monthly injections. Acta Ophthalmol (Copenh) 89: 647-653, 2011.

11. Giani A, Cigada M, Choudhry N, Deiro AP, Oldani M, Pellegrini sM, Invernizzi A, Duca P, Miller JW and Staurenghi G: Reproducibility of retinal thickness measurements on normal and pathologic eyes by different optical coherence tomography instruments. Am J Ophthalmol 150: 815-824, 2010.

12. Bentaleb-Machkour Z, Jouffroy E, Rabilloud M, Grange JD and Kodjikian L: Comparison of central macular thickness measured by three OCT models and study of interoperator variability. ScientificWorldJournal 2012: 842795, 2012.

13. Krebs I, Lie S, Stolba U, Zeiler F, Felke S and Binder S: Efficacy of intravitreal bevacizumab (Avastin) therapy for early and advanced neovascular age-related macular degeneration. Acta Ophthalmol (Copenh) 87: 611-617, 2009.

14. Martin DF, Maguire MG, Ying GS, Grunwald JE, Fine SL and Jaffe GJ; CATT Research Group: Ranibizumab and bevacizumab for neovascular age-related macular degeneration. N Engl J Med 364: 1897-1908, 2011.

15. Bashshur ZF, Haddad ZA, Schakal A, Jaafar RF, Saab M and Noureddin BN: Intravitreal bevacizumab for treatment of neovascular age-related macular degeneration: A one-year prospective study. Am J Ophthalmol 145: 249-256, 2008.
16. Francis PJ: The influence of genetics on response to treatment with ranibizumab (Lucentis) for age-related macular degeneration: the Lucentis Genotype Study (an American Ophthalmological Society thesis). Trans Am Ophthalmol Soc 109: 115-156, 2011.

17. Priya RR, Chew EY and Swaroop A: Genetic studies of age-related macular degeneration: Lessons, challenges, and opportunities for disease management. Ophthalmology 119: 2526-2536, 2012.

18. Cleary CA, Jungkim S, Ravikumar K, Kelliher C, Acheson RW and Hickey-Dwyer M: Intravitreal bevacizumab in the treatment of neovascular age-related macular degeneration, 6- and 9-month results. Eye (Lond) 22: 82-86, 2008.

19. Penn JS, Madan A, Caldwell RB, Bartoli M, Caldwell RW and Hartnett ME: Vascular endothelial growth factor in eye disease. Prog Retin Eye Res 27: 331-371, 2008.

20. Huang C, Xu Y, Li X and Wang W: Vascular endothelial growth factor A polymorphisms and age-related macular degeneration: A systematic review and meta-analysis. Mol Vis 19: 1211-1221, 2013.

21. Chopdar A, Chakravarthy U and Verma D: Age related macular degeneration. BMJ 326: 485-488, 2003.

22. Lim LS, Mitchell P, Seddon JM, Holz FG and Wong TY: Age-related macular degeneration. Lancet 379: 1728-1738, 2012

23. Coleman HR, Chan C-C, Ferris FL III and Chew EY: Age-related macular degeneration. Lancet 372: 1835-1845, 2008.

24. Levy J, Shneck M, Rosen S, Klemperer I, Rand D, Weinstein O, Pitchkhadze A, Belfair N and Lifshitz T: Intravitreal bevacizumab (avastin) for subfoveal neovascular age-related macular degeneration. Int Ophthalmol 29: 349-357, 2009.

25. Aisenbrey S, Ziemssen F, Völker M, Gelisken F, Szurman P, Jaissle G, Grisanti S and Bartz-Schmidt KU: Intravitreal bevacizumab (Avastin) for occult choroidal neovascularization in age-related macular degeneration. Graefes Arch Clin Exp Ophthalmol 245: 941-948, 2007.

26. El Matri L, Bouraoui R, Chebil A, Kort F, Bouladi M, Limaiem R and Landoulsi $\mathrm{H}$ : Bevacizumab injection in patients with age-related macular degeneration associated with poor initial visual acuity. J Ophthalmol 2012: 861384, 2012. 